\title{
Automatic guided vehicles fleet size optimization for flexible manufacturing system by grey wolf optimization algorithm
}

\author{
V. K. Chawla ${ }^{a}$, Arindam Kumar Chanda ${ }^{b}$ and Surjit Angra ${ }^{c}$
}

${ }^{a}$ Researcher, Mechanical Engineering Department ,NIT Kurukshetra, Haryana, India

${ }^{b}$ Associate Professor, Department of Mechanical and Automation Engineering, G. B. Pant Engineering College, Okhla, Delhi, India ${ }^{c}$ Professor \& Head of the Department of Mechanical Engineering, National Institute of Technology Kurukshetra, Haryana, India

\section{H R O N I C L E}

Article history:

Received: October 26, 2017

Received in revised format: November 26, 2017

Accepted: December 26, 2017

Available online:

December 26, 2017

Keywords:

Automatic Guided Vehicles

Flexible Manufacturing System

Grey wolf optimization algorithm

Fleet Size Optimization

\section{A B S T R A C T}

Automatic guided vehicle system (AGVs) plays a vital role in material handling operations for a flexible manufacturing system (FMS).Optimum AGVs fleet size selection is one of the most significant decisions in effective design and control of automated material handling system. The fleet size estimation and optimization of AGVs requires an in-depth understanding of the various factors that AGVs in the FMS relies on. In this paper, an investigation for fleet size optimization of AGVs in different layouts of FMS by application of the analytical method and grey wolf optimization algorithm (GWO) is carried out. Layout design is one of the significant factors for optimization of AGV's fleet size in any FMS. Results yield from analytical and grey wolf optimization algorithm are compared and validated for the different sizes of FMS layouts by computational experiments.

\section{Introduction}

A flexible manufacturing system (FMS) is a computer controlled manufacturing system comprising of computer controlled work centers that are capable of delivering many operations at once. FMS generally includes machining centers, automated storage-retrieval systems, automated material handling systems, automated inspection centers, etc. Automated guided vehicle (AGV) system are extensively applied in FMS for transferring semi-finished, finished jobs between the work centers and others destinations. Automated guided vehicles (AGVs) are chiefly applied in manufacturing and service industries, including automobile production plants, semiconductor fabrication shops, flexible manufacturing system (FMS), and seaport container terminals. Application of AGVs offers many benefits in comparison of traditional material handling systems. AGVs benefits include high flexibility, efficient space utilization, high shop floor safety, and easy and efficient communication with other equipment of FMS. There are many factors which are generated from real-time dynamic material transfer operations to the various components of the FMS. This makes application and evaluation of AGV systems complex and

\footnotetext{
* Corresponding author. Tel.: +919312660065

E-mail address: kumar6150046@nitkkr.ac.in (V. K. Chawla) 
critical. Estimation of the required number of AGVs and buffer capacity of AGVs are some of the critical issues to deal with (Mahadevan \& Narendran, 1990; Ganesharajah et al., 1998). AGVs fleet size estimation is one of the fundamental decisions to be made for different FMS layouts. Optimum utilization of material handling resources for work centers leads to increase in throughput of the manufacturing system and reduce earliness and tardiness in any manufacturing system. In the present study, investigations are carried out for estimation and optimization of AGVs fleet size in different FMS layout sizes by analytical model and by grey wolf optimization algorithm. The analytical method and optimization method applied are generic enough to be ported and applied to other types of layouts as well. Some significant factors are identified and included in the model in this study in which estimation of the number of AGVs largely depends on including processing time, job mix, job sequence, loading unloading station, number of work centers. AGVs fleet size is optimized after considering these factors.

The paper is presented in following sections. Section 2 presents the literature review and identifies the gap. Section 3 describes the problem statement and assumptions used along with the applied analytical model. In section 4 the grey wolf optimization algorithm is presented. Section 5 presents results yield and finally, the paper is concluded in section 6 along with possible future directions of research.

\section{Literature Review}

Automated guided vehicles (AGVs) are the crucial part of the flexible manufacturing system and different studies are carried out on AGVs considering different factors like routing, scheduling, capacity, collision avoidance and many more. The fleet size of AGVs has a high impact on the working conditions of AGV systems (Van der Meer, 2000). Installation and implementation of AGVs require highcost investments; hence optimum estimation of the fleet size of AGVs is significant from flexible manufacturing system's operations point of view. FMS with tandem guide path configuration has partitioned zones. In tandem FMS configurations the numbers of partitioned zones are equal to the number of AGVs, one partitioned zone equals to one AGV but in case of other FMS guide path configurations the required number of AGVs has to be estimated. Three prime factors were identified by Egbelu and Tanchoco (1986) which generally affect the estimation of the number of AGVs namely (1) load transfer points location (2) the guide path configuration, and (3) AGV dispatching policies or scheduling rules. Four analytical models to estimate the number of AGVs was proposed by Egbelu and Tanchoco (1986). The proposed analytical model work on the basis of the expected number of loaded trips of AGVs between the work centers and the number of work centers in the FMS facility. Egbelu and Tanchoco (1986) also signified the importance of the dispatching rules in the estimation of the fleet size of AGVs. Maxwell and Muckstadt (1982) and Mahadevan and Narendran (1994) also worked in similar directions. From the literature, it is evident that selection of appropriate dispatching rules has a crucial role in the estimation of the fleet size of AGVs. Appropriate selection and consideration of dispatching rule can yield accurate and better AGVs fleet size estimate. Srinivasan et al. (1994) considered modified FCFS rule for Multi AGVs cruising in FMS layout. Authors considered "K" number of AGVs cruising with "K" time's fast velocity. Srinivasan et al. (1994) offered good results but the same theory yield inferior solution with a high increase in requirement of AGVs. Tanchoco et al. (1987) and Talbot (2003) developed and investigated queuing model for estimation of AGV fleet size. The queuing model introduced by Talbot (2003) found to yield inferior solutions in light traffic and yields overestimation of the number of AGVs than actually required in the system. Arifin and Egbelu (2000) developed a statistical approach for estimation of AGVs. Vis et al. (2001) introduced network flow modeling for estimation of AGVs. Network flow modeling proposed by Vis et al. (2001) introduced a polynomial time minimum-flow algorithm to find an estimation of AGVs. Sinriech and Tanchoco (1992) investigated multicriteria decision modeling for estimation of AGVs fleet size in SFT layout of FMS. Sadrabadi and Sadjadi (2009) introduced a new interactive algorithm for solving multi-objective problems. The developed algorithm keeps plain continuous interaction with the decision maker and starts solution from the infeasible region and then moves step by step towards the feasible region. The developed algorithm found to be simple in nature for judgments by the decision maker and also handled its nonlinear utility 
effectively. Ji and Xia (2010) proposed and investigated an analytical model for the modern transportation requirements carried out by the automatic guided vehicles. The objective of the proposed model was to guarantee the transportation system's stability and also to minimize the AGV fleet size. Authors solved the numerical example by the proposed analytical method. Lin et al. (2010) discussed the significance of the application of the automated material handling systems for the semiconductor industry to decrease wafer cycle times and increase the fab productivity. Authors considered uncertainties of the manufacturing system and developed a simulation-optimization model to find out the optimum fleet size of the vehicles. The proposed model was validated by an empirical study. Moghadam et al. (2010) applied robust optimization to the vehicle routing problem under uncertain demands. Authors performed computational experiments to analyze the effect of the robust, the tradeoffs, and the deterministic solution. Authors applied their proposed approach in a real case study of medicine distribution and found that their investigated method reduces the uncertain demand drastically. Angeloudis and Bell (2010) investigated job assignments issues of AGVs in the container terminal settings under the uncertainty. Authors developed a generic dispatching algorithm for dispatching of the AGVs for the real-time automated container terminal operations. Yifei et al. (2010) proposed a two-step combined analytical and simulation method for estimation of AGVs requirement in the FMS facility, in which the result output of the analytical model, becomes the input of the simulation model. Authors observed the proposed procedure was to be fast and reliable. Huang et al. (2012) investigated a simulation optimization problem to allocate vehicle in $300 \mathrm{~mm}$ wafer fab. Authors conducted the discrete event simulation experiment and characterized the automatic material handling system to yield the optimum vehicle allocation for the intra-bay and inter-bay systems. To validate the results of proposed simulation method, authors solved a photo-bay example and compared the solution yield of an analytical method with the proposed simulation method.

Moghadam et al. (2012) investigated vehicle routing problem with uncertain customer demands and unknown distributions. Authors developed and applied an advanced particle swarm optimization algorithm for the solution of uncertain vehicle routing problem. A novel decoding scheme applied in advanced Particle Swarm Optimization (PSO) increased the algorithm's efficiency significantly. Authors compared results achieved from the applied algorithm with another existing algorithm to validate the proposed methodology. Choobineh et al. (2012) modeled operations of AGVs by application of multi-class queuing networks. Authors estimated AGV's fleet size under the steady-state conditions specifically for the loaded and empty-travel times; AGV's loading - unloading time and waiting time for dispatch. Further Authors validated their analytical methodology by comparing its results with the solution yield of simulation methods. Chang et al. (2014a) proposed an integrated simulation optimization and data envelopment framework for the multi-objective vehicle fleet sizing for the automated material handling system. The numerical experiments carried out by authors validated the effectiveness and better performance of the proposed framework. Chang et al. (2014b) investigated optimal fleet sizing problem by simulation sequential modeling with minimum vehicle cost under time constraints. Authors constructed a series of metamodels for an empirical study on real data to validate the viability of the simulation sequential modeling approach.

Bozorgi-Amiri et al. (2015) solved split delivery vehicle routing problem with the objective to minimize vehicle's path travel. Authors applied the memetic algorithm and considered two objective functions namely load carried by the vehicle and energy consumed by the vehicle in the transportation of the load. Authors validated the proposed approach by solving 21 standard problems and compared their solutions obtained from the mathematical programming standard solver. Gharaei et al. (2015) considered a manufacturing system operating with a unit work center on a rewards-driven system. Authors optimized the total rewards in the manufacturing system by considering the rewards in quadratic form for earliness as well as for learning. Bairagi et al. (2013) introduced TOPSIS method to Multiple Criteria Decision Making (MCDM), for evaluation, ranking, and selection of the material handling equipment (MHE). The multi-load AGVs are capable of pick and drop of additional loads which considerably 
reduce the idle AGV travel time considerably along with a reduction in distance travel. Bilge and Tanchoco (1997) carried out simulation experiment and compared the performance of unit load AGVs and multi-load AGVs. In the experiment, it was found that use of multi-load AGV increases FMS throughput significantly. Van der Meer (2000) carried out a series of simulation experiment and found that a multi-load AGVs significantly improves the FMS throughput and reduces makespan. Authors also pointed that with the application of multi-load AGVs, scheduling and dispatching becomes complex and difficult to handle.

From the literature study, it was observed that for estimation of multi-load AGVs fleet size, the application of analytical computations found to be inaccurate and does not satisfy real-time manufacturing requirements. Contrary application of simulation experiments found to be accurate and useful for estimation of multi-load AGVs fleet size. It was also observed that the estimation of the fleet size of AGVs relates to the selection of the appropriate dispatching and scheduling rules, vehicle congestion conditions, traffic management and other factors. Application of simulation models for different FMS layouts can offer an accurate estimation of the fleet size of AGVs requirement. Ho and Liu (2009) analyzed performance parameters such as throughput and tardiness for multi-load AGV systems operating under various load selection rules. Ho and Liu (2009) performed simulation tests for estimation of AGVs fleet size under pickup-dispatching rules and load-selection rules. Chawla et al. (2017) applied MMPSO algorithm and carried out computational simulation for scheduling of multi-load AGVs in FMS environment. From the study of literature, a potential research gap to estimate and optimize the AGVs fleet size in FMS by application of nature-inspired grey wolf algorithm is observed. To fill aforesaid research gap in present study an analytical model for estimation of AGVs fleet size in FMS is proposed and further, the estimated fleet size is optimized by application of grey wolf optimization algorithm in forthcoming sections of this paper.

\section{Problem Definition}

\subsection{Assumptions}

The assumptions considered are as follows,

1. A job enters into the system only through the load / unloads station and job can leave the system only through loading/unloading station.

2. There is no flow of job within the same work center.

3. Travel time between pickup and delivery workstations is deterministic.

4. Average delivery rates between the workstations are known. If there is no delivery for AGV then AGV will stay at its current position and will wait for next delivery schedule.

5. If there are multiple idle AGVs at the time of a delivery request, one empty AGV is selected by a predefined dispatching rule. If more than one delivery is waiting for an idle AGV, then the requests are taken on the first-come-first-served basis.

6. Setup time on work center for all operations is assumed to be zero.

\subsection{Problem Statement}

In this paper, all factors such as job sequence, number of work centers, total available time, volume mix, job processing time, mean spent time etc. are considered with an objective to estimate required number of AGVs by application of analytical model and further optimizing the number of AGVs required for variable size FMS facilities by application of grey wolf optimization algorithm. It is always imperative to optimize system resources to achieve the maximum throughput by maintaining the balance in system resources. AGVs are extensively applied in FMS for material handling operations and require significant investments; therefore it is highly imperative to optimize the required number of 
AGVs in FMS Mahadevan and Narendran (1990). Optimum fleet size of AGVs in FMS can lead to a potential reduction in investment of funds and increases the productivity of the entire FMS.

\subsection{Evaluation of number of AGVs-Analytical Model}

The average rate in which job enters the system is calculated on the basis of mean time spent by the jobs in the system. The mean time spent on the job is calculated by the product of all processing time and their respective probabilities summed over all possibilities. The minimum number of vehicles required in given layout can be calculated by dividing the mean time spent by each job to total time available. Initially, the required number of automated guided vehicles are calculated by applying analytical model (refer Eqs. (1-9)) considering following parameters namely job sequence, the quantity of work center, total available time, volume mix, job processing time, mean spent time etc.

In the FMS layout (Fig. 1), the load/unload station is considered as the $m+1^{\text {th }}$ work center, the flow $f_{k l}$ between any two work center $k$ and $l$ due to a unit flow at the load/unload station is given by:

\section{Notations:}

$n=$ number of jobs to be processed.

$v_{i}=$ the volume of job mix of the job $i$.

$N S E Q_{i}=$ the number of sequences for processing of job $i$

$S_{i j}=$ the probability of job $i$ processing according to the $j_{t h}$ sequence.

$N M A C_{i j}=$ the number of work centers job $i$ visit for completion of $j_{t h}$ sequence.

$P T_{i j k}=$ the processing time for job $i$ on $k_{\text {th }}$ work center processed according to the $j_{t h}$ sequence.

$t_{k l}=$ the travel time between two points $k$ and $l$.

$$
f_{k l}=\sum_{i=1}^{n} v_{i} \sum_{j=1}^{N S E Q_{i}} S_{i j} \delta_{k l} \quad \forall k, l=1, \cdots, m+1 \quad \text { and } \quad k \neq 1
$$

$\delta_{k l}=\left\{1\right.$ if work center $l_{t h}$ is visited immediately after $k$ in the $j_{t h}$ sequence of the job $i, 0$ otherwise. $\}$

$\mu=$ the property of flow matrix.

$$
\begin{aligned}
& f_{k k}=0 \quad \forall k=1, \ldots \ldots \text { to......, m+1 } \\
& \sum_{k=1}^{m} f_{m+1, k}=1 \\
& \sum_{l=1}^{m} f_{l, m+1}=1 \\
& \sum_{l=1}^{m+1} f_{k l}=\sum_{l=1}^{m+1} f_{l k} \quad \forall k=1, \cdots, m+1
\end{aligned}
$$

$\gamma_{l(i)}= \begin{cases}1 & \text { if job visits work centre } l \\ 0 & \text { if job does not visit any work centre. }\end{cases}$

$$
n \operatorname{load}_{k}=480 / \mu_{k}
$$




$$
\begin{aligned}
& f_{k l}=\sum_{i=1}^{n} v_{i} \sum_{j=1}^{N S E Q_{i}} \sum_{l=1}^{N M A C_{i j}}\left[\frac{\left\{v_{i} S_{i j} \gamma_{k(i j)}\right\}}{\sum_{i=1}^{n} \sum_{j=1}^{N S E Q_{i}} v_{i} S_{i j} \gamma_{k(i j)}}\right] \times P T_{i j k} \quad \forall k=1, \cdots, m \\
& \mathrm{TR}=\alpha \times\left\{\sum_{k=1}^{m+1} n \operatorname{load}_{k} \times\left[\sum_{k=1}^{m+1} p_{k l}\left(t_{k b}+t_{k l}+l_{k}+u_{b}+l_{b}+u_{l}\right)\right]\right\}+(1-\alpha) \times\left\{\sum_{k=1}^{m+1} n \operatorname{load} \times\left[\sum_{k=1}^{m+1} p_{k l}\left(t_{k l}+l_{k}+u_{l}\right)\right]\right\} \\
& N=\frac{T R}{T A}
\end{aligned}
$$

The transition probability matrix $\mathrm{P}=\left[\mathrm{P}_{k}\right]$ is obtained by normalizing the process matrix $\mathrm{F}$ such that the row sum is 1 means the probability always remains between 0 and 1 .

$\mu_{k}=$ average rate at which work center $k$ processes the jobs

where

$$
\gamma_{k(i j)}= \begin{cases}1 & 1 \text { if machine } k \text { is visited in the } j t h \text { sequence of job } i \\ 0 & \text { otherwise. }\end{cases}
$$

Let $\alpha=$ how many times jobs are routed through the central buffer in case of less storage in front of work centres.

Where,

$T R=$ Total time available for all AGVs.

$T A=$ Time available per AGV.

Due to less storage in front of the work centres sometimes jobs are routed through the central buffer.

Let $\alpha$ time this happens hence total time $(T R)$ is calculated and time available per AGV $(T A)$ is calculated. With the division of $T R$ and $T A$, the required number of AGVs can be estimated.

\section{Grey wolf optimization algorithm}

\subsection{AGVs fleet size optimization}

AGVs fleet size optimization is carried out by application of grey wolf optimization algorithm considering all factors, parameters, and equations same as considered above in the analytical model. While applying grey wolf optimization algorithm all parameters are tuned further to optimize the fleet size of AGVs in aforementioned FMS layout.

\subsection{Methodology of Grey wolf optimization algorithm}

Grey wolf optimization algorithm is based on leadership hierarchy and intelligent hunting process carried out by the grey wolves. For simulating the social hierarchy, four types of grey wolves: alpha, beta, delta, and omega are considered. In grey wolf optimization algorithm main steps include searching and encircling the prey, attacking the prey and then hunting the prey Mirzalili et al. (2014), Tung et al. (2015) and Mirzalili et al. (2016). In the optimization process, four fitness functions are considered namely: alpha, beta, gamma, and omega. The survival of the fittest solution is considered as alpha $(\alpha)$.Thus, the second and third best solutions are termed as beta $(\beta)$ and delta $(\Delta)$, respectively. The 
remaining candidate solution is considered as Omega ( $\omega$ ). Mirzalili et al. (2014), Mirzalili et al. (2016) and Bozorg-Haddad (2017). The encircling behavior is given by the following equation:

$$
\begin{aligned}
& \vec{D}=\left|\vec{C} \cdot \vec{X}_{p}(t)-\vec{X}(t)\right| \\
& \vec{X}(t+1)=\vec{X}_{p}(t)-\vec{A} \cdot \vec{D}
\end{aligned}
$$

where,

$\mathrm{t}=$ current iteration number,

$\overrightarrow{\mathrm{A}}$ and $\overrightarrow{\mathrm{C}}=$ coefficients vectors of the bootstrap program, and

$\vec{X}_{p}(t)=$ Prey position vector.

$\overrightarrow{\mathrm{X}}=$ Vector of the grey wolf's positions.

$\overrightarrow{\mathrm{D}}=$ Calculated vector used to specify a new position of the grey wolf.

$\overrightarrow{\mathrm{A}}=2 \vec{a} \cdot \overrightarrow{\mathrm{r}}_{1}-\overrightarrow{\mathrm{a}}$

$\overrightarrow{\mathrm{C}}=2 \overrightarrow{\mathrm{r}}_{2}$

$\overrightarrow{\mathrm{a}}=$ Vector set to decrease linearly from 2 to 0 over the iterations.

$\overrightarrow{\mathrm{r}}_{1}$ and $\overrightarrow{\mathrm{r}}_{2}=$ random vectors in $[0,1]$.

Grey wolf at (x,y) position can change and update its position according to prey position (x', y'). The best agent can be placed with relative to their present position by controlling $\vec{A}$ and $\vec{C}$. The hunting mechanism of grey wolves can be simulated by considering that wolves keep on attacking till the prey stops its movements. The hunting behavior of grey wolves can be modeled and simulated by considering the value of $\vec{a}$ decreasing in different iterations and the fluctuation rate of $\vec{A}$ also decreases. We assume that alpha ( $\alpha$, best agent) beta $(\beta)$ and delta $(\Delta)$ has more information about the prey position. So the algorithm stores the three best solutions and after that, it initiates Omega $(\omega)$, the fourth one to update its position so as to achieve best position in search space which further optimizes the results. Alpha $\alpha$, Beta $(\beta)$ and Delta $(\Delta)$ estimate the prey's location and other wolves such as Omega $(\omega)$ update their position randomly around the victim. The process of grey wolf optimizing algorithm is also shown in Fig. 1 as the flowchart of grey wolf optimisation algorithm. The grey wolf optimization algorithm's hunting mechanism can be modeled as follows.

$$
\begin{aligned}
& \overrightarrow{\mathrm{D}}_{\alpha}=\left|\overrightarrow{\mathrm{C}}_{1} \cdot \overrightarrow{\mathrm{X}}_{\alpha}-\overrightarrow{\mathrm{X}}\right| \\
& \overrightarrow{\mathrm{D}}_{\beta}=\left|\overrightarrow{\mathrm{C}}_{2} \cdot \overrightarrow{\mathrm{X}}_{\beta}-\overrightarrow{\mathrm{X}}\right| \\
& \overrightarrow{\mathrm{D}}_{\Delta}=\left|\overrightarrow{\mathrm{C}}_{3} \cdot \overrightarrow{\mathrm{X}}_{\Delta}-\overrightarrow{\mathrm{X}}\right| \\
& \overrightarrow{\mathrm{X}}_{1}=\overrightarrow{\mathrm{X}}_{\alpha}-\mathrm{A}_{1} \cdot\left(\overrightarrow{\mathrm{D}}_{\alpha}\right) \\
& \overrightarrow{\mathrm{X}}_{2}=\overrightarrow{\mathrm{X}}_{\beta}-\mathrm{A}_{2} \cdot\left(\overrightarrow{\mathrm{D}}_{\beta}\right) \\
& \overrightarrow{\mathrm{X}}_{3}=\overrightarrow{\mathrm{X}}_{\Delta}-\mathrm{A}_{3} \cdot\left(\overrightarrow{\mathrm{D}}_{\Delta}\right) \\
& \overrightarrow{\mathrm{X}}(\mathrm{t}+1)=(\overrightarrow{\mathrm{X}} 1+\overrightarrow{\mathrm{X}} 2+\overrightarrow{\mathrm{X}} 3) / 3
\end{aligned}
$$

The grey wolf optimization algorithm exhibits following advantages over other metaheuristic techniques.

1. Easy to implement due to a simple structure.

2. Less storage required than other technique.

3. Convergence is faster due to continuous reduction of search space and decision variables. 


\section{Experimental Results}

The analytical model and grey wolf optimization algorithm are programmed in Matlab for three different FMS layouts. The three FMS layouts are shown below in Figs. (3-5). Further result yield from the program in terms of AGV fleet size are compared for analytical as well as for grey wolf optimization algorithm. Pseudocode of grey wolf optimization algorithm is portrayed below in Fig. 2. In Fig. 3 the FMS layout is comprised of 6 number of work centres and one loading and unloading centre, in Fig. 4 FMS layout consists of 8 number of work centres and one loading and unloading centre and in Fig. 5 the FMS layout constitutes of 11 work centres along with one loading and unloading centre.

\section{Table 1}

$\underline{\text { Result comparison of analytical model and grey wolf optimization algorithm. }}$

\begin{tabular}{|c|c|c|c|c|c|}
\hline \multirow{2}{*}{ FMS Layout } & \multirow{2}{*}{ No. of Jobs } & \multirow{2}{*}{ No. of Work centres } & \multirow{2}{*}{ No. of Sequences } & \multicolumn{2}{|c|}{ No. of AGVs } \\
\hline & & & & Analytical & GWO \\
\hline 1 & 5 & 6 & 2 & 2 & 1 \\
\hline 2 & 5 & 8 & 2 & 4 & 2 \\
\hline 3 & 5 & 11 & 2 & 5 & 3 \\
\hline
\end{tabular}

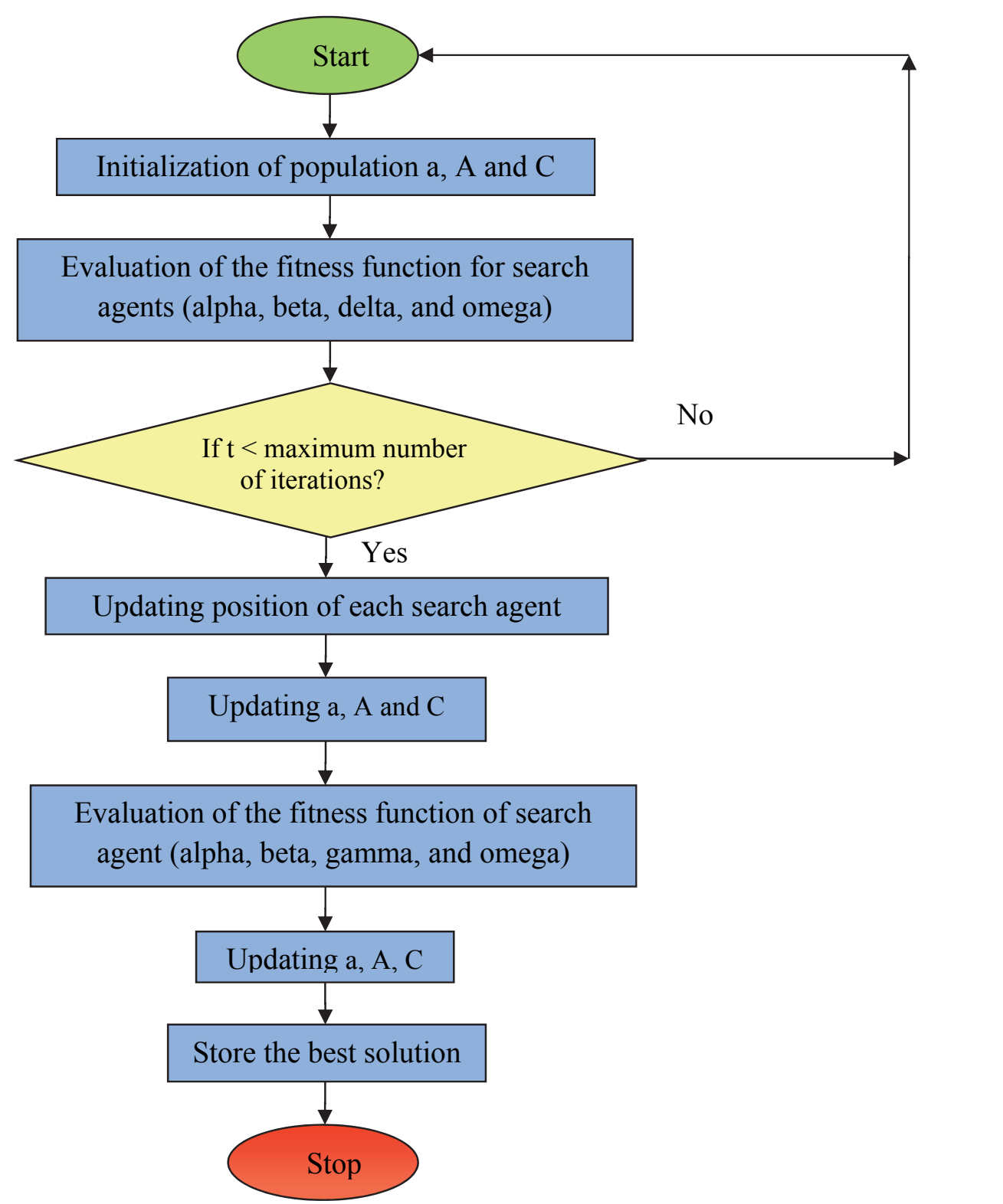

Fig. 1. Flowchart of grey wolf optimization algorithm. 


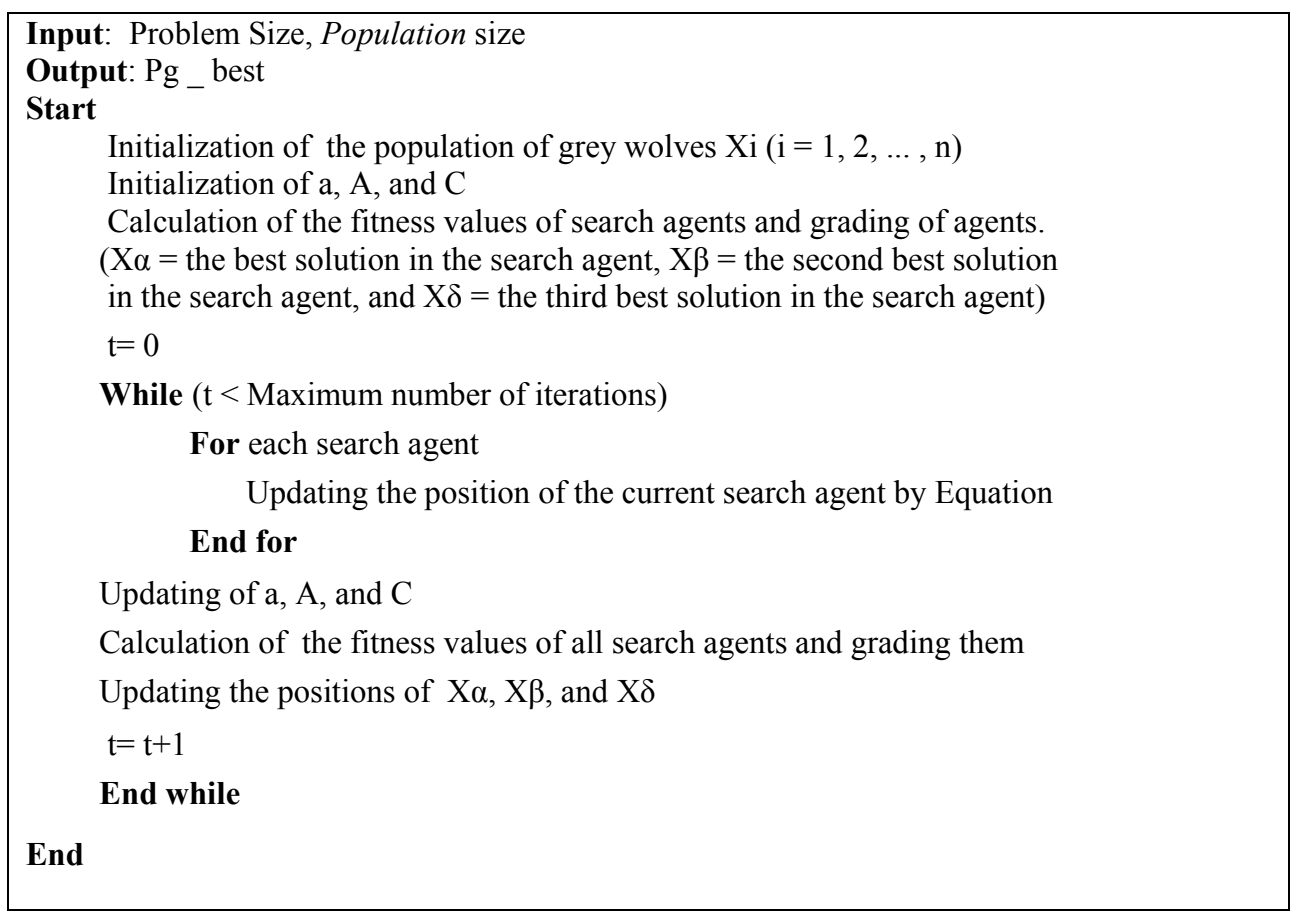

Fig. 2. Pseudocode of grey wolf optimization algorithm.

The results obtained from the analytical model for FMS layout 1,2 and 3 constituting of 6,8 and 11 work centers along with one loading and unloading center each yield 2, 4 and 5 numbers of AGVs respectively. Application of grey wolf optimization algorithm optimized the fleet size of AGVs to 1, 2 and 3 numbers for FMS layout 1,2 and 3 constituting of 6,8 and 11 work centers along with one loading and unloading center respectively.

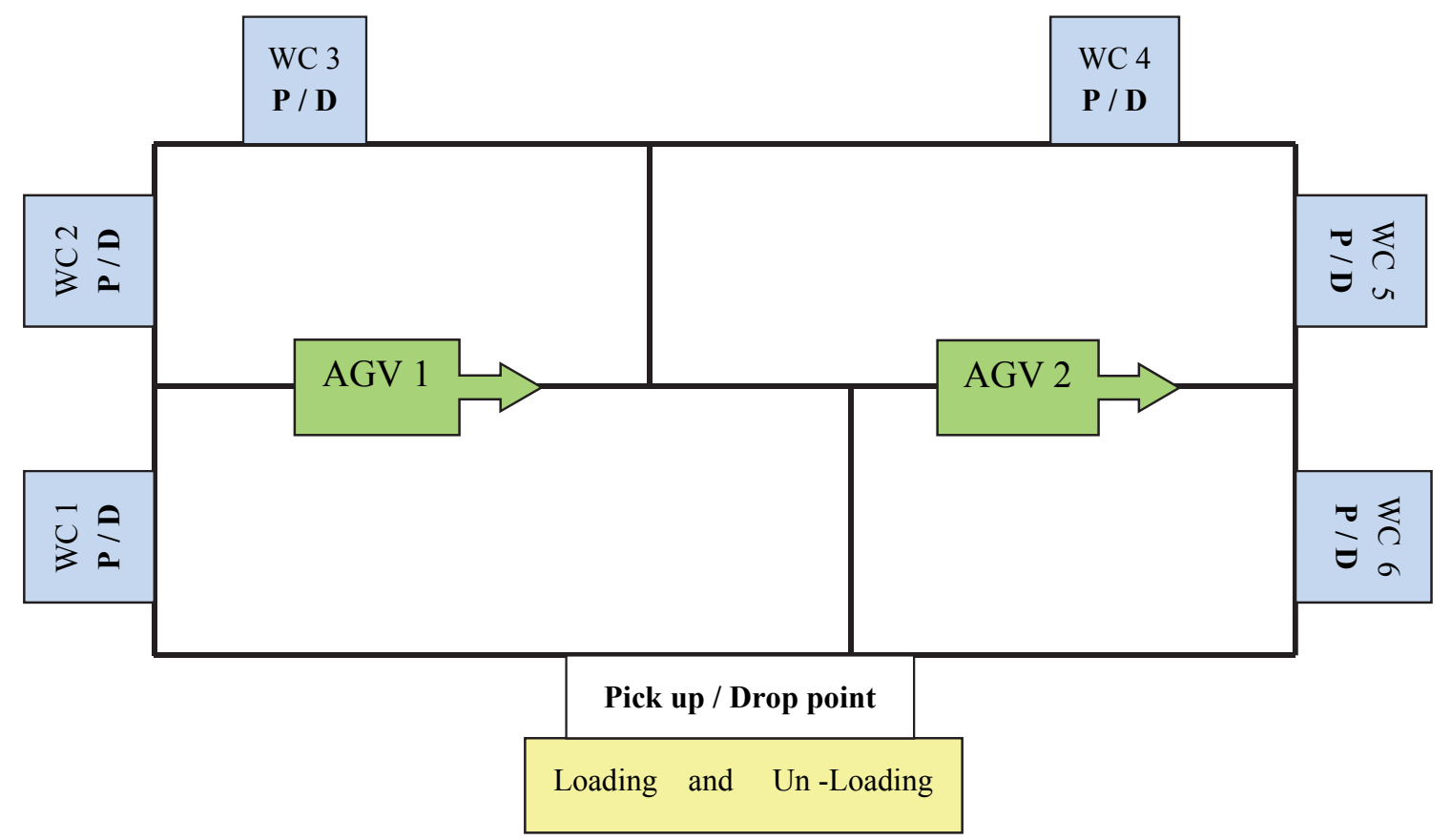

Fig. 3 The FMS layout 1 comprised of 6 numbers of work center and one loading and unloading center.

The Grey wolf algorithm is run for 100 iterations and the population size of the grey wolves is taken 30. Parameter adjustment of grey wolf algorithm for above problem is carried out by considering the 
two random vectors $\overrightarrow{\mathrm{r}}_{1}$ and $\overrightarrow{\mathrm{r}}_{2}$ and the controlling parameter $\overrightarrow{\mathrm{a}}$. In the simulation run of GWO algorithm, the two random vectors $\vec{r}_{1}$ and $\vec{r}_{2}$ are considered in the range of $(0,1)$ and the controlling parameter $\vec{a}$ considered to have linearly decreasing values from 2 to 0 during the iterations. All simulation run is carried out by MATLAB software on a computer with an Intel(R) Core(TM) i5 processor. Results yield of the analytical model and GWO algorithm are mentioned in Table 1 and comparison of the same is presented in form of a graph in Fig. 6.

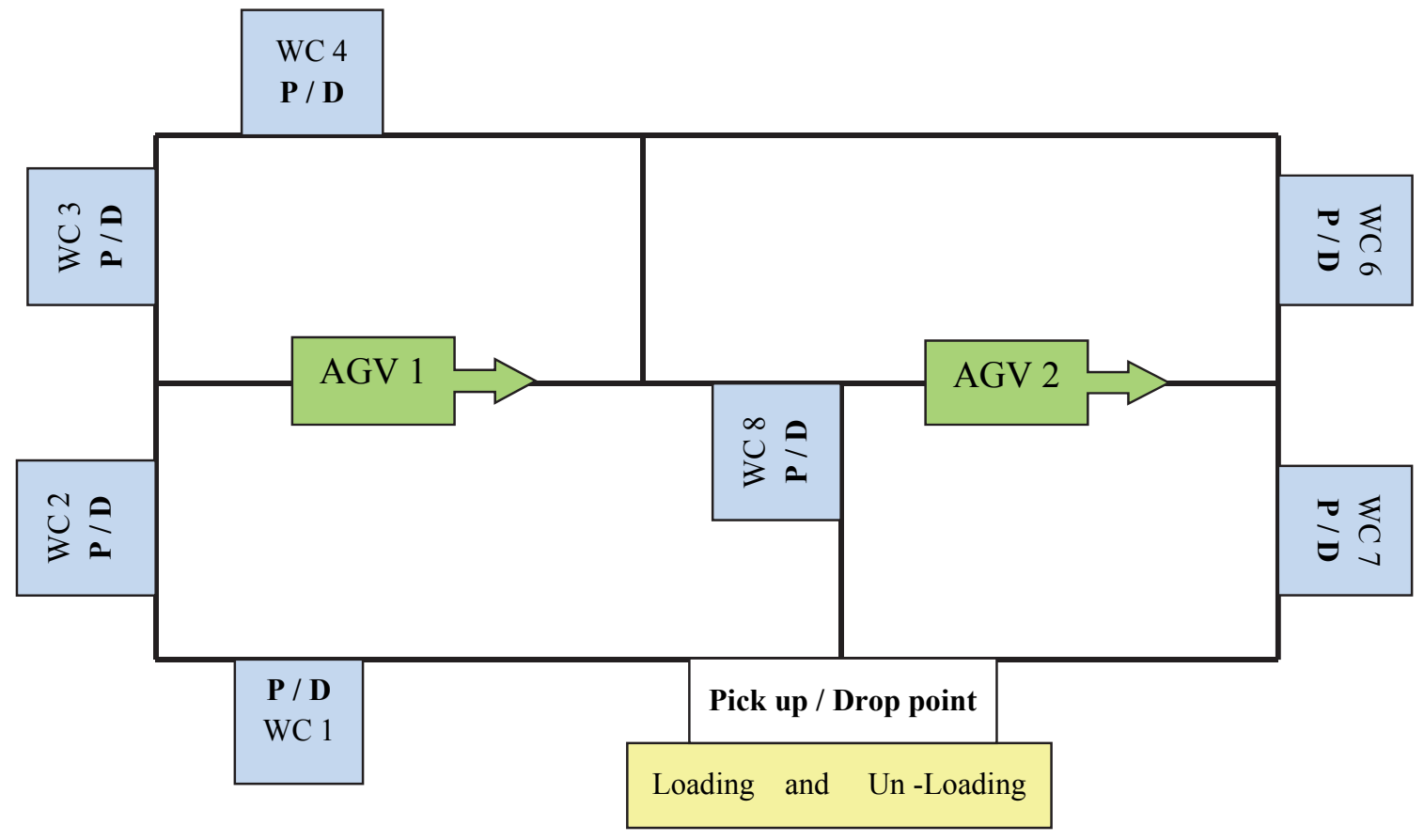

Fig. 4. The FMS layout 2 consisting of 8 numbers of work center and one loading and unloading center.

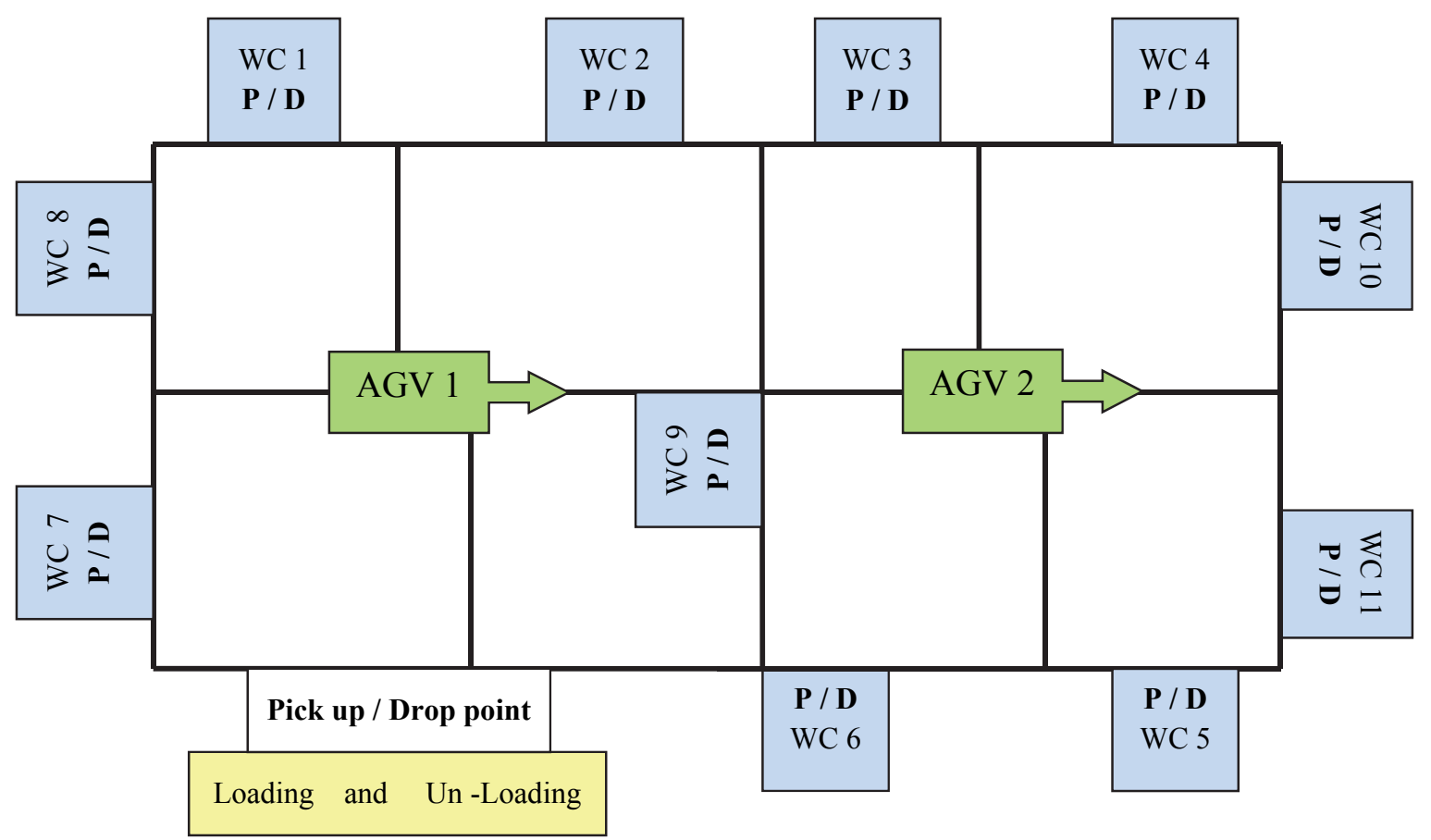

Fig. 5. The FMS layout 3 constituting 11 numbers of work center and one loading and unloading center. 


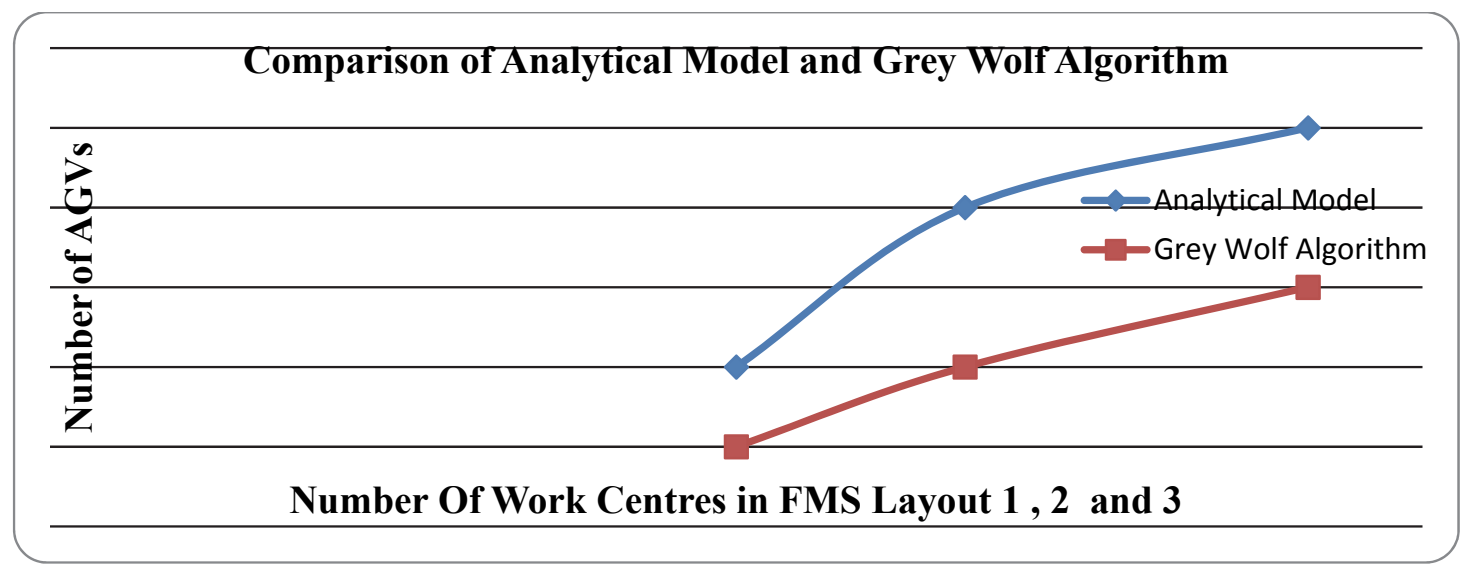

Fig. 6. Comparison of analytical and grey wolf optimization algorithm for different FMS layouts.

\section{Conclusion and Future Work}

In the present paper, Fleet size of AGVs for varying size of FMS layouts have been calculated by analytical model and further, the fleet size of AGVs are optimized by grey wolf optimization algorithm computationally on Matlab software. Three different sizes of FMS layouts were considered with 6, 8 and 11 numbers of work centers along with one loading and unloading center each. In evaluating and optimizing the fleet size of AGVs, various design parameters e.g. processing time, type of job, job flow, type of sequence etc. were considered. From the results, it is clear that grey wolf optimization algorithm outperforms calculated the fleet size of AGVs by the analytical model and further optimize the required number of AGVs to 1,2 and 3 numbers for three different FMS layouts consisting of 6,8 and 11 numbers of work centers respectively.

Future research directions may be carried out for AGV fleet size calculation and optimization by application of genetic algorithm, particle swarm optimization algorithm or other hybrid algorithms considering other critical operating factors of FMS and AGVs such as dispatching or scheduling rules, factor of reliability of work centres in FMS and AGVs, AGVs cruising speed, type of AGVs i.e. unit load, multi-load, uni-directional, bi-directional etc.

\section{Acknowledgement}

The authors would like to express their sincere thanks to the anonymous reviewers for their valuable observations and comments for an earlier version of this research paper.

\section{References}

Angeloudis, P., \& Bell, M. G. (2010). An uncertainty-aware AGV assignment algorithm for automated container terminals. Transportation Research Part E: Logistics and Transportation Review, 46(3), 354-366.

Arifin, R., \& Egbelu, P. J. (2000). Determination of vehicle requirements in automated guided vehicle systems: a statistical approach. Production Planning \& Control, 11(3), 258-270.

Bairagi, B., Dey, B., \& Sarkar, B. (2013). Incremental analysis for the performance evaluation of material handling equipment: A holistic approach. Uncertain Supply Chain Management, 1(2), 77-86.

Bilge, U., \& Tanchoco, J. M. (1997). AGV systems with multi-load carriers: basic issues and potential benefits. Journal of Manufacturing Systems, 16(3), 159.

Bozorgi-Amiri, A., Mahmoodian, V., Fahimnia, E., \& Saffari, H. (2015). A new memetic algorithm for solving split delivery vehicle routing problem. Management Science Letters, 5(11), 1017-1022.

Bozorg-Haddad, O. (2017). Advanced Optimization by Nature-Inspired Algorithms.

Chang, K. H., Chang, A. L., \& Kuo, C. Y. (2014a). A simulation-based framework for multi-objective vehicle fleet sizing of automated material handling systems: an empirical study. Journal of Simulation, 8(4), 271-280. 
Chang, K. H., Huang, Y. H., \& Yang, S. P. (2014b). Vehicle fleet sizing for automated material handling systems to minimize cost subject to time constraints. IIE Transactions, 46(3), 301-312.

Chawla, V.K., Chanda, A.K., \& Angra, S. (2017). Scheduling of multi-load AGVs in FMS by modified memetic particle swarm optimization algorithm. Journal of Project Management, 3(1), 39-54.

Choobineh, F. F., Asef-Vaziri, A., \& Huang, X. (2012). Fleet sizing of automated guided vehicles: a linear programming approach based on closed queuing networks. International Journal of Production Research, 50(12), $3222-3235$.

Egbelu, P. J., \& Tanchoco, J. M. A. (1986). Potentials for bi-directional guide-path for automated guided vehiclebased systems. International Journal of Production Research, 24(5), 1075-1097.

Ganesharajah, T., Hall, N. G., \& Sriskandarajah, C. (1998). Design and operational issues in AGV-served manufacturing systems. Annals of Operations Research, 76, 109-154.

Gharaei, A., Naderi, B., \& Mohammadi, M. (2015). Optimization of rewards in single work center scheduling in the rewards-driven systems. Management Science Letters, 5(6), 629-638.

Ho, Y. C., \& Liu, H. C. (2009). The performance of load-selection rules and pickup-dispatching rules for multipleload AGVs. Journal of Manufacturing Systems, 28(1), 1-10.

Huang, C. J., Chang, K. H., \& Lin, J. T. (2012). Optimal vehicle allocation for an automated materials handling system using simulation optimisation. International Journal of Production Research, 50(20), 5734-5746.

Ji, M., \& Xia, J. (2010). Analysis of vehicle requirements in a general automated guided vehicle system based transportation system. Computers \& Industrial Engineering, 59(4), 544-551.

Lin, J. T., Chang, K. H., \& Huang, C. J. (2010, October). Dynamic vehicle allocation in automated material handling system. In Industrial Engineering and Engineering Management (IE\&EM), 2010

IEEE 17Th International Conference on (pp. 1523-1527). IEEE.

Mahadevan, B., \& T. T. Narendran. (1990). Design of an automated guided vehicle-based material handling system for a flexible manufacturing system. The International Journal of Production Research 28(9), 1611-1622.

Mahadevan, B., \& Narendran, T. T. (1994). A hybrid modeling approach to the design of an AGV-based material handling system for an FMS. The International Journal of Production Research, 32(9), 2015-2030.

Maxwell, W. L., \& Muckstadt, J. A. (1982). Design of automatic guided vehicle systems. IIE Transactions, 14(2), 114-124.

Moghadam, B. F., Sadjadi, S. J., \& Seyedhosseini, S. M. (2010). An empirical analysis on robust vehicle routing problem: a case study on drug industry. International Journal of Logistics Systems and Management, 7(4), 507518.

Moghaddam, B. F., Ruiz, R., \& Sadjadi, S. J. (2012). Vehicle routing problem with uncertain demands: An advanced particle swarm algorithm. Computers \& Industrial Engineering, 62(1), 306-317.

Sadrabadi, M. R., \& Sadjadi, S. J. (2009). A new approach to solve multiple objective programming problems. International Journal of Industrial Engineering \& Production Research, 20(1), 41-51.

Sinriech, D., \& Tanchoco, J. M. A. (1992). Impact of empty vehicle flow on performance of single-loop AGV systems. The International Journal of Production Research, 30(10), 2237-2252.

Srinivasan, M. M., Bozer, Y. A., \& Cho, M. (1994). Trip-based material handling systems: throughput capacity analysis. IIE Transactions, 26(1), 70-89.

Talbot, L. (2003). Design and performance analysis of multi-station automated guided vehicle systems (Doctoral dissertation, Université Catholique de Louvain).

Tanchoco, J. M. A., Egbelu, P. J., \& Taghaboni, F. (1987). Determination of the total number of vehicles in an AGVbased material transport system. Material Flow, 4(1-2), 33-51.

Tung, N.S., \& Chakravorty, S. (2015). Grey Wolf optimization for active power dispatch planning problem considering generator constraints and valve point effect. International Journal of Hybrid Information Technology 8(12), 117-134.

Van der Meer, R. (2000). Operational control of internal transport (No. TTS; T2000/5).

Vis, I. F., De Koster, R. M. B. M., Roodbergen, K. J., \& Peeters, L. W. (2001). Determination of the number of automated guided vehicles required at a semi-automated container terminal. Journal of the Operational research Society, 409-417.

Yifei, T., Junruo, C., Meihong, L., Xianxi, L., \& Yali, F. (2010, July). An estimate and simulation approach to determining the automated guided vehicle fleet size in FMS. In Computer Science and Information Technology (ICCSIT), 2010 3rd IEEE International Conference on (Vol. 9, pp. 432-435). IEEE.

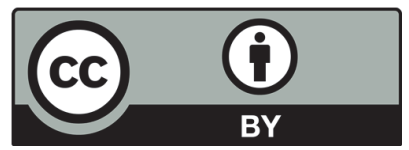

(C) 2018 by the authors; licensee Growing Science, Canada. This is an open access article distributed under the terms and conditions of the Creative Commons Attribution (CC-BY) license (http://creativecommons.org/licenses/by/4.0/). 